УДК: 622.276.76: 622. 244.7.

DOI: $10.15587 / 2313-8416.2015 .37216$

\title{
ИССЛЕДОВАНИЕ СОБСТВЕННЫХ КОЛЕБАНИЙ ПЕРЕДВИЖНОГО АГРЕГАТА ПРИ ПРОЕЗДЕ ПО ГРУНТОВОЙ ДОРОГЕ
}

\section{(C) М. И. Казымов}

Передвижной подъемный агрегат для выполнения спускоподъемных работ часто проезжая по грунтовым дорогам совериает свободные колебания. Эти колебания приводят к волнистому виду поверхности дороги. А это вредит чувствительности аппаратов и оборудования, находящегося на кузове агрегата. Поэтому определение характеристик собственных колебаний агрегата является актуальным вопросом. Для определения частоты собственных колебаний агрегата задача сведена к определению площуди контакта двух соприкасающихся цุилиндров

Ключевые слова: передвижной агрегат, неровности дороги, свободные колебания, частота колебания, соприкасающиеся изилиндры

The mobile lifting aggregate for carrying out round-trip operations often passing through earth road performs free oscillations. These oscillations cause formation of washboard road surface. And this damage the sensitivity of mechanisms and equipment set on the aggregate body. Hence, the determination of aggregate self-oscillation is an actual issue. To determine the aggregate self-oscillation frequency the task is reduced to determining contact square of two adjacent cylinders

Keywords: mobile aggregate, road inequalities, self-oscillation, oscillation frequency, contact cylinders

\section{1. Введение}

После проезда неровностей, передвижной агрегат (КОРО 80/100 и 4АН-700 и др.) над ровной поверхностью совершает свободные колебания. Собственные частоты и коэффициенты затухания, характеризующие эти колебания, оказывают существенное влияние на поведения агрегата и на образование новых неровностей на ровной части дороги. При неоднократном повторение колебательного движения поверхность грунтовой дороги получает волнистую форму. Когда частота этих волн совпадает с собственной частотой, при колебаний агрегата возникает резонанс, что приводит к увеличению амплитуды колебаний кузова передвижного агрегата, на котором находятся чувствительные приборы. Увеличение амплитуд колебаний может привести к повреждению этих приборов и самого агрегата.

\section{3. Цель работы}

Целью данной работы является исследование собственных частот и периода колебаний агрегата. Если частоту и период собственных колебаний обозначить соответственно через $n$ и $T$ скорость автомобиля через $v_{0}$, то расстояние между впадинами на дороге, которые образуются за счет колебаний автомобиля, будет равно

$$
l_{0}=T \cdot v_{0}
$$

Известно что:

$$
T=\frac{1}{n}
$$

В работе [1] для $n$ получено выражение

$$
n=\frac{1}{2 \pi} \sqrt{\frac{g}{f}} c e k^{-1}
$$

где $f$ - прогиб упругих элементов.
4. Исследование собственных колебаний передвижного агрегата

Для определения $f$ рассмотрим задачу об определении площади контакта двух соприкасающихся цилиндров. В частном случае, когда дорога ровная, радиус внешнего цилиндра будет бесконечность.

Два цилиндра с радиусами $R_{1}$ и $R_{2}$ соприкасаются в точке о. Систему координат выберем как па рис. 1.

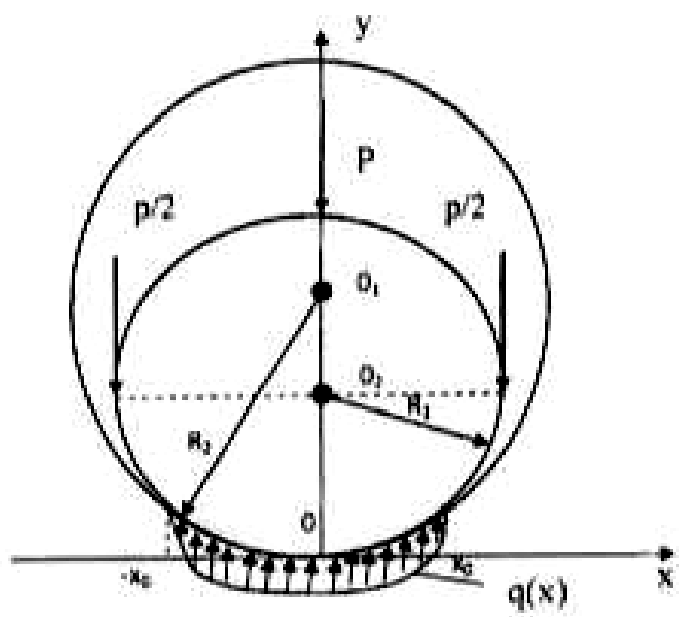

Рис. 1. Соприкасание двух цилиндров

Внутренний цилиндр прижимается к внешнему цилиндру силой $\mathrm{P}$, за счет чего внутренний цилиндр деформируясь приобретает овальный вид и образуется контактная площадь, абсциссы крайних точек которой обозначим через $-x_{0}$ и $x_{0}$. Со стороны внешнего цилиндра на внутренний цилиндр действует распределенная сила, интенсивность которой обозначим через $q(x)$. Если 
провести мысленное горизонтальное сечение, проходящее через центр внутреннего цилиндра, то в точках сечения будут действовать сила $\mathrm{P} / 2$. Если моделировать нижнюю половину внутреннего цилиндра как кривая упругая балка, то уравнение изогнутой оси балки будет:

$$
\frac{1}{R_{2}}-\frac{1}{R_{1}}=\frac{M_{u 3}}{E_{2} I_{z_{2}}}
$$

где $R_{1}$ и $R_{2}$ - радиусы внутреннего и внешнего цилиндров соответственно; $E_{1}$ - модуль юнга материла внутреннего цилиндра; $I_{z_{2}}$ - момент инерции поперечного сечения балки относительно оси $Z$, которая перпендикулярна к плоскости $O X Y$ и проходит через точку 0. $M_{\text {из }}-$ изгибающий момент. Зазор между цилиндрами бесконечно малая величина, поэтому будем считать, что все точки балки получают вертикальные перемещения.

Уравнения окружностей до деформации будут:

$$
\begin{aligned}
& \left.\begin{array}{lll}
x^{2} & \left(y_{1}\right. & R_{1}
\end{array}\right)^{2} \quad R_{1}^{2}, \\
& \left.\begin{array}{lll}
x^{2} & \left(y_{2}\right. & R_{2}
\end{array}\right)^{2} \quad R_{2}^{2} \text {. }
\end{aligned}
$$

Из (2)

$$
\begin{aligned}
& y_{1}=R_{1}-\sqrt{R_{1}^{2}-x^{2}} ; \\
& -y_{2}=R_{2}-\sqrt{R_{2}^{2}-x^{2}} .
\end{aligned}
$$

Тогда перемещения точек внутреннего цилиндра на участке контакта будут:

$$
y \quad y_{1} \quad y_{2} \quad R_{1} \quad R_{2} \quad \sqrt{R_{1}^{2} x^{2}} \quad \sqrt{R_{2}^{2} x^{2}},
$$

где $x$ меняется от $-x_{0}$ до $x_{0}$ т.е. $x \in\left(-x_{0} ; x_{0}\right)$

Из-за того, что зазор между цилиндрами бесконечно малым, перемещение $y$ также будет малым т.е. можно считать, что

$$
\frac{1}{R_{2}} \quad \frac{1}{R_{1}} \quad y .
$$

Тогда

$$
\begin{aligned}
& M_{\text {из }}(\mathrm{x}) \quad \mathrm{E}_{2} J_{z_{2}} y, \\
& Q(x) \quad \mathrm{E}_{2} J_{z_{2}} y, \\
& q \quad \mathrm{E}_{2} J_{z_{2}} y^{I V},
\end{aligned}
$$

где $Q(x)$ - перерезывающая сила

$$
\text { Вычислим } y_{(x)}^{I V} \text {. Из (7) }
$$

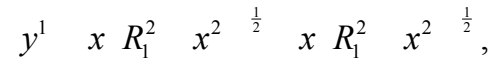

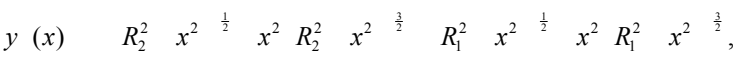

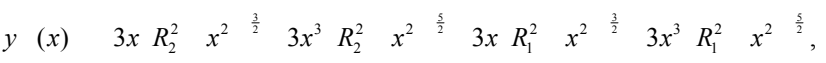

$$
\begin{aligned}
& y^{I V}(x) \quad 3 R_{2}^{2} \quad x^{2} \frac{3}{2} \quad 18 x^{2} \quad R_{2}^{2} \quad x^{2} \frac{5}{2}
\end{aligned}
$$

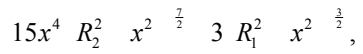

$$
-18 x^{2}\left(R_{1}^{2}-x^{2}\right)^{-\frac{5}{2}}+15 x^{4}\left(R_{1}^{2}-x^{2}\right)^{-\frac{7}{2}} .
$$

Подставляя (16) в (9) имеем

$$
\begin{array}{ccccccccc}
q(x) & E_{1} J_{z_{2}} & 3 & R_{1}^{2} & x^{2} & & R_{2}^{2} & x^{2} & \frac{3}{2} \\
18 x^{2} & R_{1}^{2} & x^{2} & \frac{5}{2} & R_{2}^{2} & x^{2} & \frac{5}{2} & \\
15 x^{4} & R_{1}^{2} & x^{2} & & R_{2}^{2} & x^{2} & &
\end{array}
$$

Из условия равновесия имеем:

$$
{ }_{x_{0}}^{x_{0}} q(x) d x \quad P .
$$$$
P .
$$

Учитывая, что граница интегрирования симметричная и $q(x)$-функция четная из (10) имеем

$$
\int_{-x_{0}}^{x_{0}} q(x) d x=-\frac{P}{2}
$$

Функция $P(x)=y^{\prime \prime \prime}(x)$ является первобытной функцией $f(x)=y^{I V}(x)$ поэтому из (14) получаем:

$$
\begin{aligned}
& x_{0}\left[\left(R_{1}^{2}-x_{0}^{2}\right)^{-\frac{3}{2}}-\left(R_{2}^{2}-x_{0}^{2}\right)^{-\frac{3}{2}}\right]+ \\
& +x_{0}^{3}\left[\left(R_{1}^{2}-x_{0}^{2}\right)^{-\frac{5}{2}}-\left(R_{2}^{2}-x_{0}^{2}\right)^{-\frac{5}{2}}\right]=-\frac{P}{6 E_{1} J_{z_{2}}}
\end{aligned}
$$

или

$$
\begin{aligned}
& \frac{x_{0}}{R_{1}^{2} \quad x_{0}^{2} \sqrt{R_{1}^{2} \quad x_{0}^{2}}} \frac{x_{0}}{R_{2}^{2} x_{0}^{2} \sqrt{R_{2}^{2} x_{0}^{2}}} \frac{x_{0}^{3}}{R_{1}^{2} x_{0}^{2} \sqrt{R_{1}^{2} x_{0}^{2}}} \\
& \frac{x_{0}^{3}}{R_{2}^{2} \quad x_{0}^{2} \sqrt{R_{2}^{2} x_{0}^{2}}} \quad \frac{P}{6 E_{2} J_{z_{2}}} \text {. }
\end{aligned}
$$

Для малых значений $P \frac{x_{0}}{R_{1}}<<1, \frac{x_{0}}{R_{2}}<<1$, тогда из (15), пренебрегая малыми значениями членов имеем: $\frac{x_{0}}{R_{2}^{3}}-\frac{x_{0}}{R_{1}^{3}}=\frac{P}{6 E_{2} J_{z_{2}}}$, откуда:

$$
x_{0}=\frac{P R_{1}^{3} R_{2}^{3}}{6\left(R_{1}^{3}-R_{2}^{3}\right) E_{2} J_{z_{2}}}
$$

для малых значений $x_{0}$ длина контактной части $l_{p} \approx 2 x_{0}$ и из (16)

$$
l_{1} \frac{P R_{1}^{3} R_{2}^{3}}{3 R_{1}^{2} \quad R_{2}^{2} \quad E_{2} J_{z_{2}}} .
$$

В случае большой сжимающей силы $P, x_{0}$ определяется из (16) численным путем. Если считать, что внутренний цилиндр абсолютно жесткий внешний цилиндр деформируется и длина контактной части будет. 


$$
l_{2} \frac{P R_{1}^{3} R_{2}^{3}}{3 R_{1}^{3} \quad R_{2}^{3} E_{2} J_{z_{2}}}
$$

где $J_{z_{1}}$ - осевой момент инерции, Е $\mathrm{E}_{2}$-модуль юнга материала внешнего цилиндра. В случае, когда деформируются оба цилиндра длина контактной части будет.

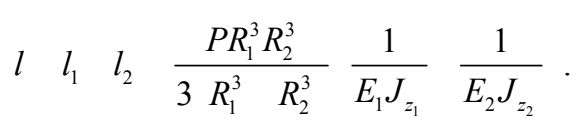

Если применять результаты уравнений (16)(19), для нашего случая, то $R_{1}-$ радиус колеса автомобиля, $R_{2}$ радиус впадины на дороге, $E_{1}-$ жесткость покрышки, $J_{z_{1}}$ - момент инерции колеса, $E_{z_{2}}$ - момент инерции грунта и дороги, $P$ - сила приложения на одно колесо. Учитывая, что момент инерции грунта бесконечно большой, то можно считать, что $J_{z_{2}}=0$. Тогда формула (19) получает вид:

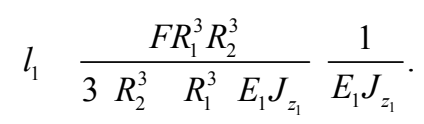

В частном случае когда дорога ровная, $R=\infty$ и равенство (20) получает вид:

$$
l_{2} \frac{F R_{1}^{3} R_{2}^{3}}{3 R_{2}^{3} 1 \frac{R_{1}^{3}}{R_{2}^{3}}} \frac{1}{E_{1} J_{z_{1}}} \frac{F R_{1}^{3}}{E_{1} J_{z_{1}}} .
$$

Учитывая, что перемещение, определяемое равенством (7) получает максимальное значение при $x=x_{0}$ а $x_{0}=l_{2}$, то подставляя (20) в (7) находим $y_{\max }$ которая является прогибом упругого элемента т.е колеса. Тогда $l=y_{\max }$ и подставляя выражение для $y_{\max }$ в (3) находим частоту собственных колебаний автомобиля.

Что касается определения жесткости $E_{1}$, то об этом есть достаточно большая информация в существующей литературе.

\section{5. Выводы}

1. В результате проведенных исследований установлено, что передвижные, агрегаты, проезжая по грунтовым дорогами, совершают свободные колебания и эти колебания приводят поверхность дороги к волнистому виду.

2. Для определения характеристики частоты собственных колебаний передвижного агрегата задача сведена к определению площади контакта двух соприкасающихся цилиндров.

3. Получено выражение для определения длины контактной части цилиндров для равной части дороги

4. Получено выражение для определения максимальное значение прогиба упругого элемента (колеса) через кого которой определяется частоты собственных колебаний передвижного агрегата.

\section{Литературы}

1. Силаев, А. А. Спектральная теория подрессоривания транспортных машин [Текст] / А. А. Силаев. Изд. Машиностроение», 1972. - 355 с.

2. Амензаде, Ю. А. Теория упругости [Текст] / Ю. А. Амензаде. - М.: «Высшая школа», 1976. - 272 с.

3. Феодосьев, В. И. Сопротивление материалов [Текст] / В. И. Феодосьев. - М.: Наука, 1970. - 544 с.

4. Мусхелишвили, Н. И. Некоторые задачи математической теории упругости [Текст] / Н. И. Мусхелишвили. - М. Наука, 1966. - 648 с.

5. Тарг, С. М. Краткий курс теоретической механики [Текст] / С. М. Тарг. - М. Физматиз, 1968. - 480 с.

6. Беляев, Н. М. Сопротивление материалов [Текст] / Н. М. Беляев. - М. Наука, 1963. - 856 с.

\section{References}

1. Silaev, A. A. (1972). Spektral'naja teorija podressorivanija transportnyh mashin. Izd. Mashinostroenie», 355.

2. Amenzade, Ju. A. (1976). Teorija uprugosti. Moscow: «Vysshaja shkola», 272.

3. Feodos'ev, V. I. (1970). Soprotivlenie materialov. Moscow: Nauka, 544.

4. Mushelishvili, N. I. (1966). Nekotorye zadachi matematicheskoj teorii uprugosti. Moscow: Nauka, 648.

5. Targ, S. M. (1968). Kratkij kurs teoreticheskoj mehaniki. Moscow: Fizmatiz, 480.

6. Beljaev, N. M. (1963). Soprotivlenie materialov. Moscow: Nauka, 856.

Рекомендовано до публікаиії д-р техн. наук, професор Гасанова Р. А. Дата надходження рукопису 23.01.2015

Казымов Муса Исмаил оглы, кандидат технических наук, доцент, кафедра «Прикладная механика», Азербайджанская Государственная Нефтяная Академия, пр. Азадлыг, 20, г. Баку, Азейрбаджан, AZ1010 E-mail: qezale@mail.ru 